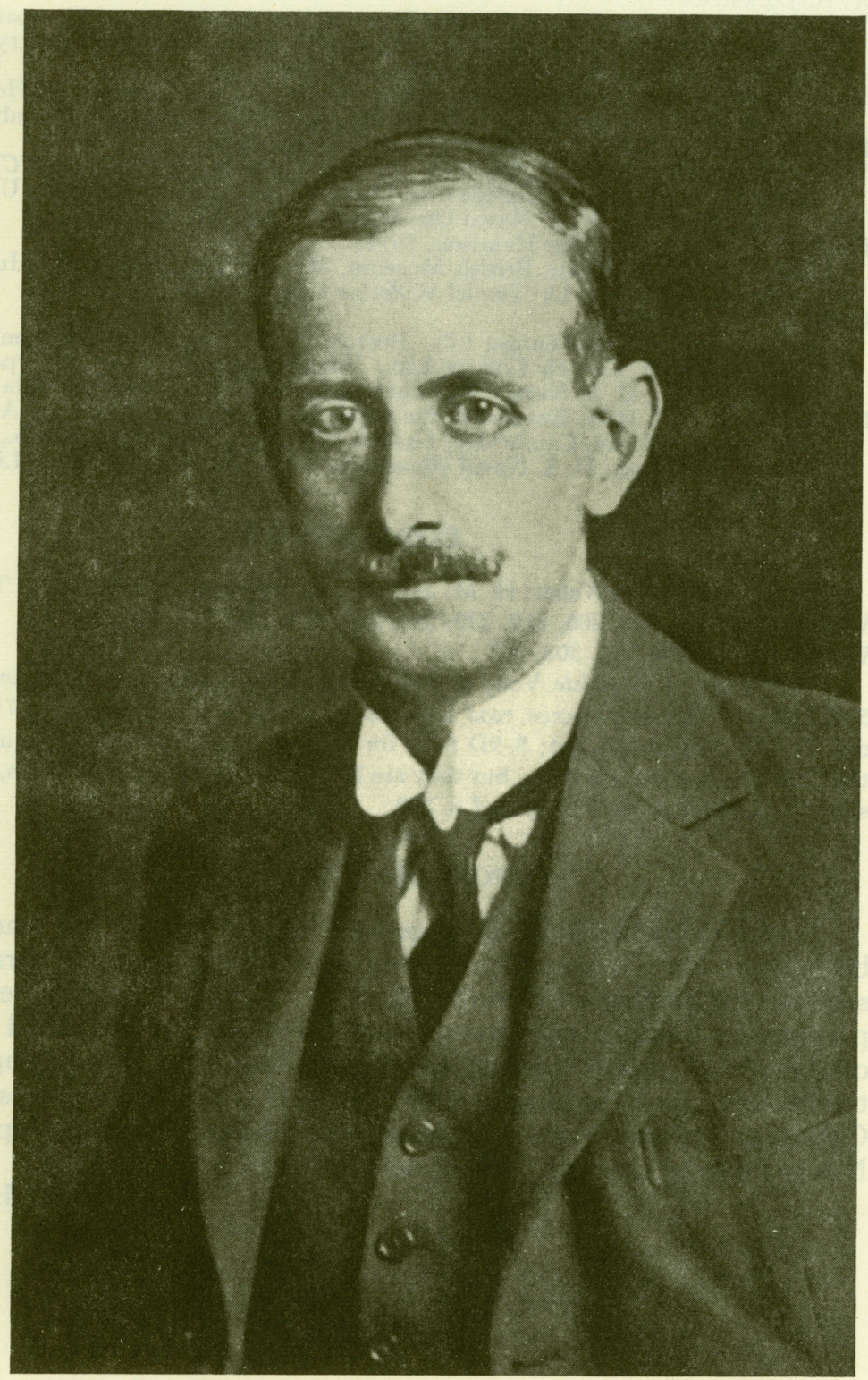

G. N. WATSON 1934 


\section{The California Institute of Technology Electric Analog Computer}

Introduction. The California Institute of Technology Analog Computer has been developed to provide a general-purpose device having as wide a field of application as is considered practicable using the electric analog principle. The vast majority of problems in the general field of engineering analysis usually require no more than a one per cent accuracy in their solutions. With this lenient accuracy requirement, many simplifications can be made in the computer and its operation. Electrical resistance, inductance, capacitance, and transformer circuits can be used to simulate the linear terms of both algebraic and differential equations. ${ }^{1-7}$ This choice of circuits is in contrast to other electric analog computers, such as REAC (Reeves Electronic Analog Computer), which employ electronic amplifiers for all integration and differentiation operations. The electronic analog computers can provide higher accuracy and can be used as simulators on a one-to-one time base, but they are more restricted in their range of application than computers of the type discussed here.

With electric analog computers only one independent variable can be continuously represented. However, by the use of finite-difference methods, such as those employed with digital computers, more independent variables can be handled. The computer at the California Institute of Technology is designed to handle linear partial differential equations with up to three independent variables and non-linear partial differential equations with two independent variables. Two basic circuit techniques are employed. In the first, a single independent variable is represented continuously as time on the computer. ${ }^{2,15,18}$ In the other, discrete values of several independent variables are represented in a form suitable for the application of finitedifference methods; in this case, the analogous circuit becomes a steadystate a-c or d-c mesh. ${ }^{15}$ Both techniques are applicable to both ordinary and partial differential equations. More complex electronic equipment is required in the application of the first principle. It involves devices to generate suitable functions of the independent and dependent variables, multipliers for multiplying any two variables together, devices for simulating arbitrary functions of a dependent variable, and amplifiers to provide the proper power output loads from these devices and to produce negative impedance terms and the unilateral or unsymmetrical terms of a matrix or servosystem.

Description of the Computer. In July 1947 the Institute's computer was placed in active service although it was only partially completed. ${ }^{1-3}$ Since then it has been in full-time use for the solution of commercial engineering problems and for Institute research. The computer is best described by referring to Fig. 1 and Table $^{21}$ which show its general layout and list the component parts. Its design embodies 110 separate sets of precision inductors, resistors, and capacitors, and 25 special transformers. These circuit elements are used to set up circuits for solving ordinary or partial differential equations. The inductors have a " $Q$ " of better than one hundred under all conditions of operation, and every element can be set to within one per cent. 


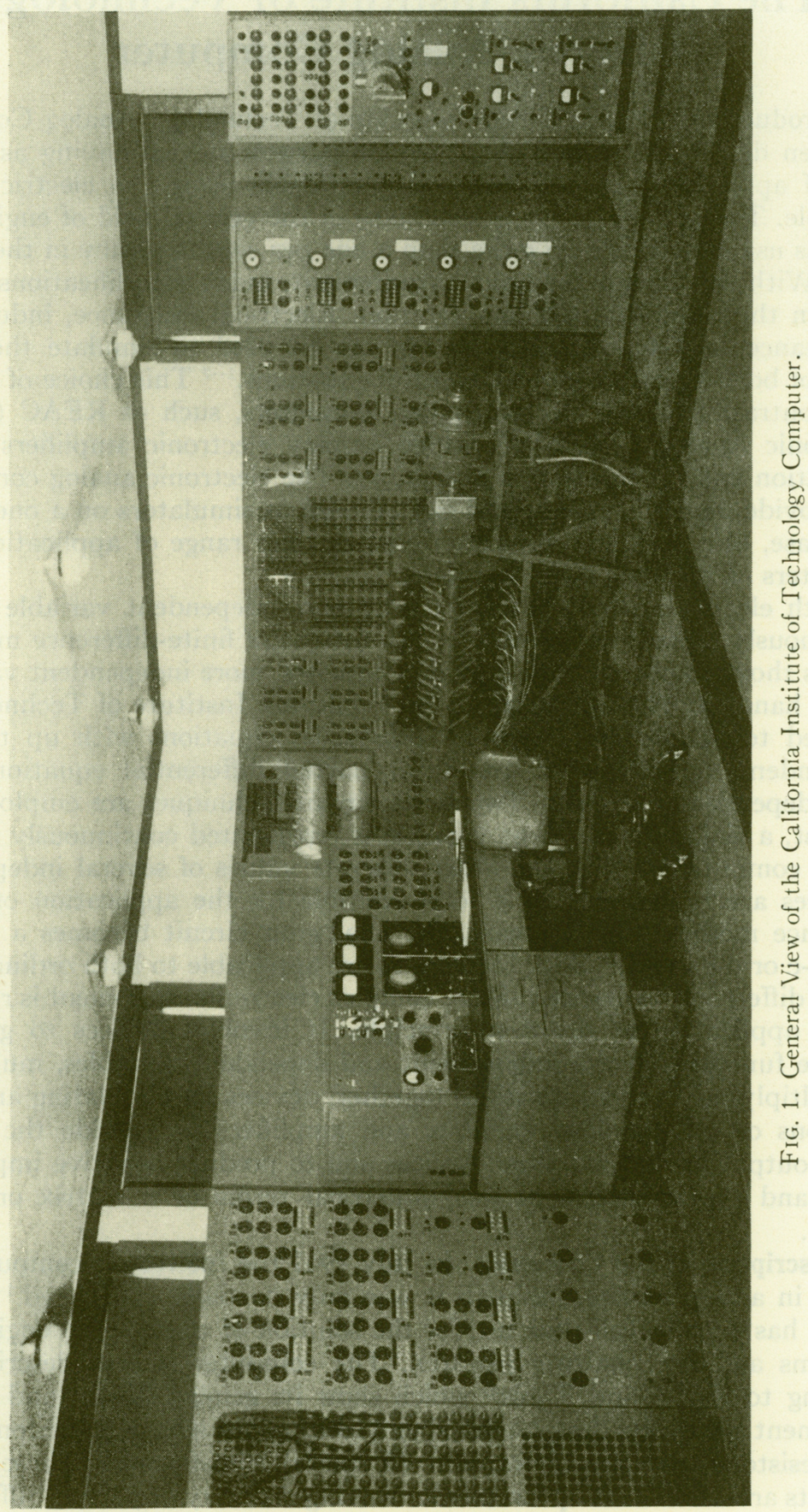




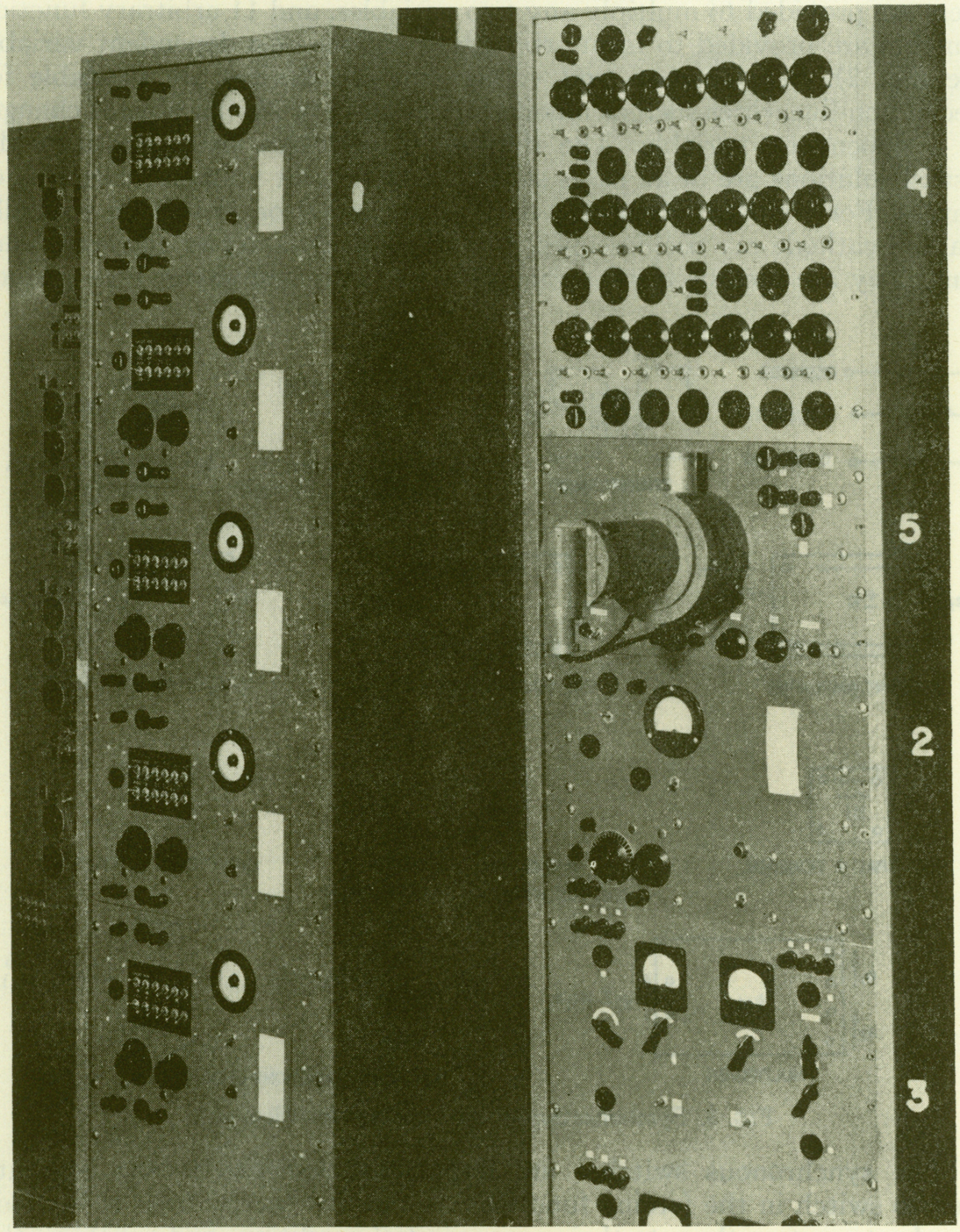

FiG. 2. Photograph of Amplifiers and Arbitrary Function Generators.

1. Negative gain servo-amplifiers.

2. Positive gain (d-c) amplifiers.

3 . Voltage and current limiters.

4. Dependent variable functions generator-Type I.

5. Dependent variable functions generator-Type II.

There are, at present, 32 separate forcing functions for introducing (as known voltages, currents, or charge) the known functions of the independent variable. These may be steady-state, variable-frequency, sinusoidal functions; square wave transient functions; or perfectly arbitrary functions of time. As listed in the Table, 20 amplifiers are now available to represent negative impedance terms and unilateral terms such as the unsymmetrical terms of a matrix. For simulating the non-linear terms of an equation, ten multi- 
pliers are provided to multiply any two variables, and 11 arbitrary function elements are provided to form arbitrary functions of a dependent variable (see Fig. 2). Five current- and voltage-limiting devices are available for special non-linearities such as missile rudder stops or similar abrupt changes on a dependent variable. The dependent variables that constitute the desired solutions are in all cases simulated by circuit voltages, currents, or charges. For transient solutions, they are recorded or viewed on specially adapted cathode-ray oscilloscopes, while for steady-state or algebraic solutions, they are measured with vacuum-tube or dynamometer-type meters.
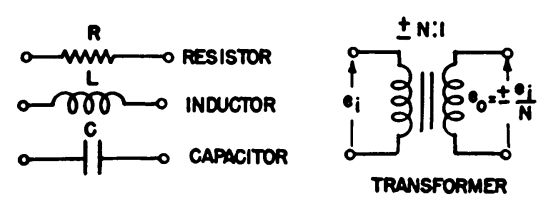

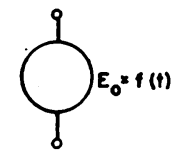

ARBITRARY FUNCTON OF INDEPENDENT VARIABLE

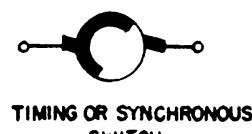
SWITCH

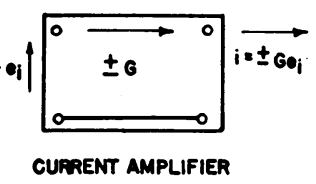

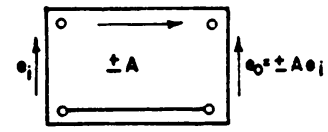

D.C. VOTAAEE AMPUFIER

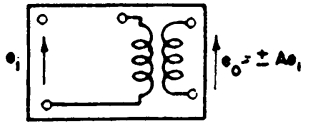

AC. POWER AMPUFIER

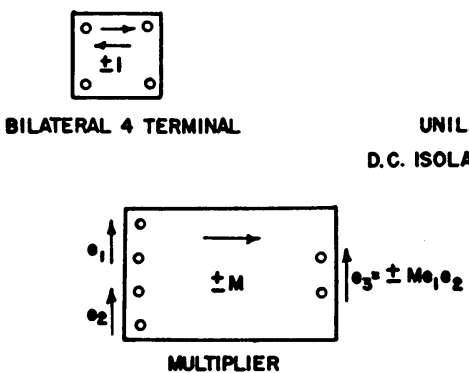

$$
\begin{array}{ll}
0 & \pm_{A} \\
0 & 0
\end{array}
$$

UNILATERAL 4 TERMINAL

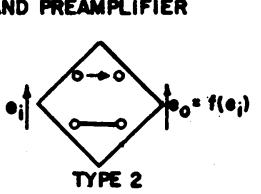

AREITRARY FUNCTIONS OF DE PENDENT UMRLALE.

FIG. 3. Symbolization of Basic Computer Elements.

The synchronous switches are important elements of the computer used to switch in the forcing functions, to remove energy from the circuits in order to restore them to their proper initial condition, and to make other sudden changes in circuit conditions. Thirty-six of these switches are now available. They are required only for transient solutions, which may be obtained in either of two ways. One way is to apply the transient just once and record it photographically or with some other memory device such as magnetic tape. The other way is to apply the transient cyclically (usually ten times a second) so that the solution appears as a steady image on a cathode-ray oscilloscope. In both techniques, each switch can be very accurately timed for opening and closing operations. Other important features of this analog computer are the plug-board arrangement and the metering circuits which permit quick connection and rapid metering of all solutions. ${ }^{6}$ Proper voltages or currents can be readily inserted at the boundaries or mesh points of circuits which simulate difference-equation approximations to partial differential equations. 
A special effort was made to design all elements of the computer so that additions can be readily made if sufficiently important problems arise that require greater facilities.

Examples of Computer Techniques. The required specifications for the analog elements are best considered by first illustrating their application to specific simple problems and describing the analog techniques employed. The basic analog elements are listed in Fig. 3 in terms of the symbols employed for circuit layouts. Typical analogous circuits for both ordinary and partial differential equations are given in Figs. 4 to 7 . Figs. 4 to 6 describe

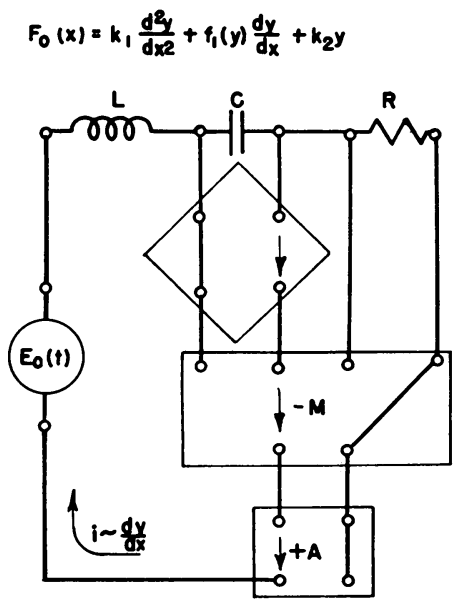

ANALOGOUS CIRCUIT EOUATION:

$E_{0}(t)=L \frac{d^{2} q}{d t^{2}}+\frac{A M R}{C} F_{1}(q) \cdot \frac{d q}{d t}+\frac{q}{C}$

( $R \frac{\mathrm{dg}}{\mathrm{d}}$ NEGUGIBLE)

MUMERICAL CONVERSION FORMULA:

$y=a \frac{F_{0}}{E_{0}} q ; L=\frac{a}{n^{2}} k_{1} ; \quad \frac{1}{C} a k_{2} ; \frac{A M R}{C} \times F_{1}(q) \frac{O}{1} f_{1}(q)$

WHERE $O$ a IMPEDANCE bASE CHANGE FACTOR

AND $n$ = INDEPENDENT VARLABLE CONVERSION FACTOR

(ONE UNIT OF $x$ IN ITS SYSTEM OF UNITS EQULL $\frac{1}{n}$ UNITS

OF $\uparrow$ ' IN SECONDS. I

F1G. 4. Illustrative Analogy for Ordinary Differential Equation.

techniques in which time continuously represents one independent variable on the computer. Fig. 7 illustrates the solution of partial differential equations with a steady-state network, a very useful iterative method of solving many non-linear partial differential equations. ${ }^{15}$

Fig. 4 illustrates the basic technique of forming an equation by one or both of KIRCHHOFF's circuit laws, the use of inductors and resistors for linear differential terms, and the use of both the multiplier and non-linear function devices for non-linear terms. Fig. 5 shows the use of transformers for mutual bilateral terms and the isolator and amplifier for unilateral or non-symmetrical terms of a matrix of simultaneous equations. 


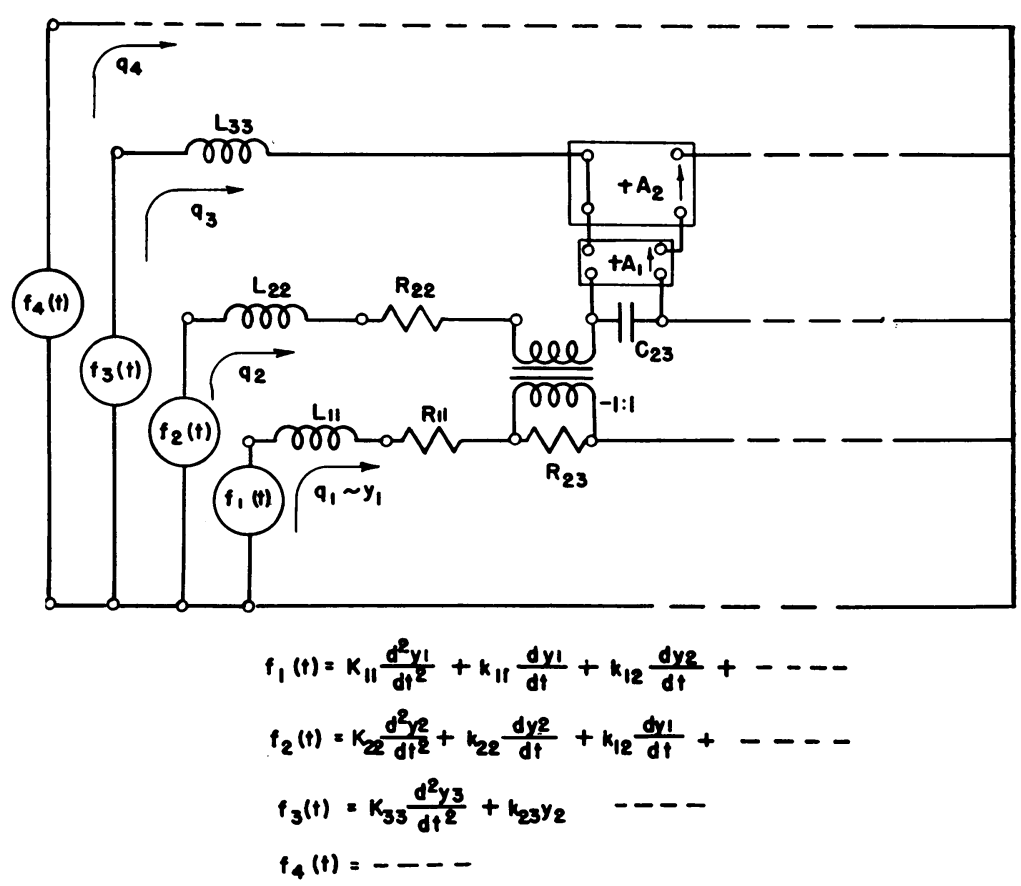

FIG. 5. Illustrative Analogy for Simultaneous Equations.

In Fig. 6, a partial differential equation is considered which requires multipliers and represents one of the independent variables continuously as time on the computer. This technique has limited application to nonlinear partial differential equations, since too many special electronic elements would be required for general application.

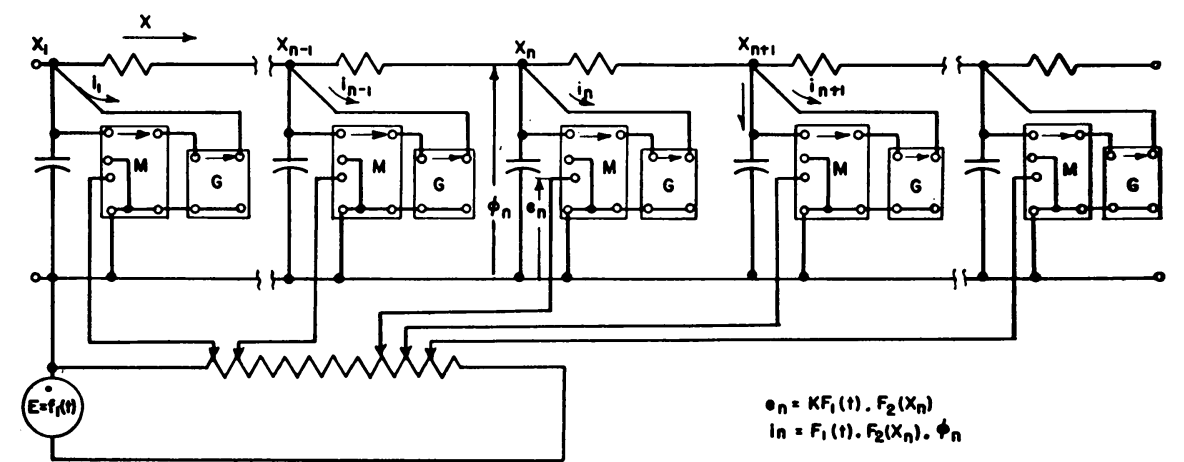

FINTE DIFFERENCE EOUATION:

$$
\frac{\phi_{n+1}-\phi_{n}}{\Delta x \Delta x_{n}} \frac{\phi_{n}-\phi_{n-1}}{\Delta x \Delta x_{n-1}}=k \frac{\partial \phi_{0}}{\partial t}+F_{1}(t) \cdot F_{2}\left(x_{n}\right) \cdot \phi_{n}
$$

COMPLETE TIME ANALOGY FOR FARTIAL DIFFERENTIAL EQUATION OF HEAT TRAMSFER.

$$
\frac{\partial e \phi}{\partial x}=k \frac{\partial \phi}{d t}+F_{1}(t) . F_{2}(x) . \phi
$$

Fig. 6. Complete Time Analogy for Partial Differential Equation of Heat Transfer. 
The general technique illustrated in Fig. 7 for non-linear partial differential equations has so far been found to have a surprisingly wide field of application to equations containing terms up to the fourth order of differentiation and to pairs of simultaneous equations. Since circuit element settings and inserted mesh currents can be changed rapidly, iterative methods of solution can be set up in a very short time.
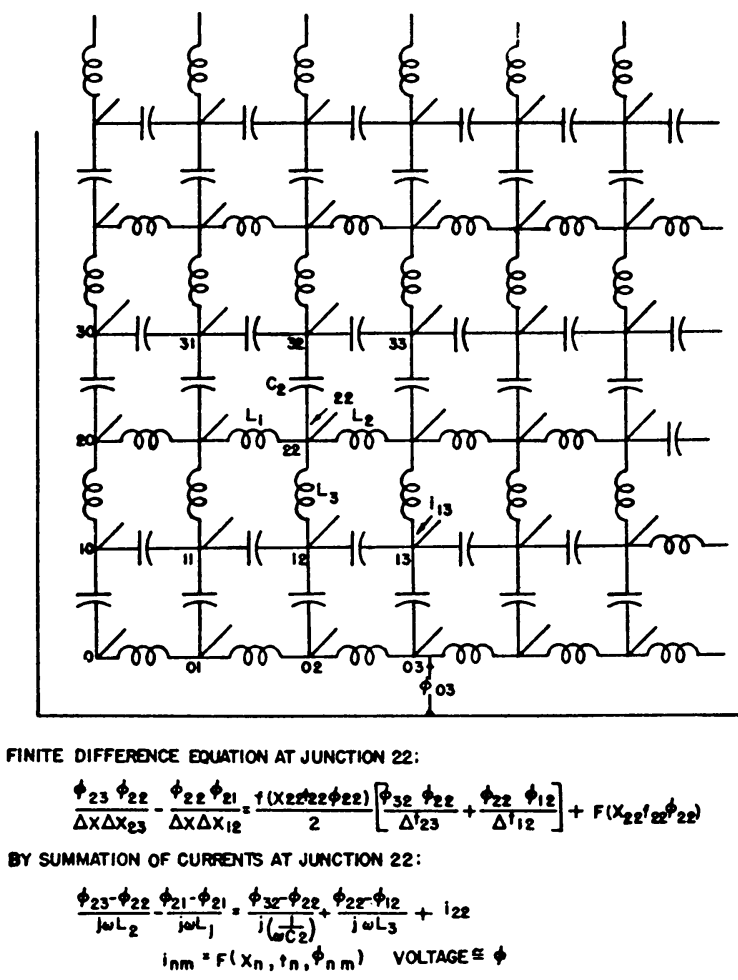

ANALOCOUS CIRCUIT FOR NON-LINEAR PARTIAL DIFFERENTIAL EOUATIONS OF THE FORM $\frac{\partial^{2} \phi}{\partial x^{2}}=1(x, t, \phi) \frac{\partial \phi}{\partial t}+f(x, t, \phi)$ WTH TIME SIMULLATED AS A SFACE VARIABLE.

Fig. 7.

As illustrated in the examples, RLC circuit elements are usually employed for all linear self-impedance terms. They are also employed for bilateral mutual terms, with junction points between the meshes simulating the simultaneous equations, and for transfer functions. Special transformers ${ }^{5}$ are used, wherever possible, for bilateral mutuals requiring circuit isolation and, in conjunction with amplifiers, to provide a four-terminal unilateral element. The RLC circuit elements set the speed of computation. In order to have the elements behave as near-ideal resistors, inductors, and capacitors, the natural frequencies of the analog circuits must lie within certain limits. The normal operating frequency range was chosen to be from ten cycles to one or two kilocycles per second.1, 6 Over this wide frequency range, transformers have certain limitations that require the occasional use of 
"isolators" to make them operate properly from zero frequency (d-c) up to the limit of one or two kilocycles per second (see Fig. 3).

The circuit impedance base must be kept low and the power level reasonably high to minimize stray coupling and stray pickup problems and enable the quick set-up of a wide variety of circuits. Unintentional impedances, such as the output impedance of a voltage amplifier, must be kept below a few ohms in series elements and above several hundred thousand ohms (or in some cases 10 or 20 megohms) in the case of shunt impedances. It is difficult to keep the ratio of the series-to-magnetizing impedance of a transformer sufficiently low over such a wide operating frequency range. This, together with the tendency of transformer coupled feedback circuits to "motor-boat," constitute the major difficulties encountered in the use of transformers. The "motor-boating" is only important when transient solutions are being obtained by repeating cyclically the forcing functions at the basic 10-cycle-per-second repetition rate in order to obtain a standing-wave solution on an oscilloscope. It can be overcome by applying the transient just once and recording the solution photographically or with a magnetic-tape recorder.

All of the electronic equipment is required to have less than one per cent amplitude distortion throughout the operating range. Furthermore, the frequency response of the equipment must not vary more than .1 decibel throughout the frequency range of the computer. The response is considered to be satisfactory if the phase shift is less than one degree throughout the entire frequency range. However, since these devices are frequently used in cascade and feedback circuits, thie phase and amplitude characteristics must be carefully controlled to eliminate oscillations at both high and low frequencies. To prevent low frequency "motor-boating," all electronic equipment except the initial forcing-function generators was designed for operation from zero frequency $(d-c)$ to the upper frequency limit of the computer. The high-frequency limit of all equipment was extended as far as practicable to make it easier to eliminate high-frequency oscillations.

The electronic elements discussed above have been described in detail. ${ }^{7,17}$ It might be of interest, however, to discuss briefly the circuit elements for non-linear functions.

Generator of an Arbitrary Function of the Dependent Variable. One of the generators of non-linear functions of the dependent variable (Type 1) uses crystal diodes and batteries in the manner shown in Fig. 8. The adjustable resistors are relatively large compared to the forward impedance of the crystals, so that the variations of this impedance with current do not affect the over-all impedance appreciably. If the switches are all thrown to the left in Fig. 8, each parallel branch will not conduct current until the terminal voltage rises above the battery voltage of that branch. The resulting current-voltage characteristic is composed of a series of straight lines, and the slope of successive segments decreases as the voltage rises. Such a characteristic is shown in curves A of Fig. 8. The two curves labeled A are both of the same characteristic, but they indicate that both the positive and negative parts of a function may have entirely different characteristics if desired.

If the switches are thrown to the right, a different characteristic results. Each branch produces a circulating current which passes through $R_{0}$. 
The battery voltage $E_{0}$ gives zero terminal voltage when no external current enters the terminals. For a given applied voltage, the current is determined by the effective resistance of all the branches in parallel. As soon as the terminal voltage exceeds the lowest branch battery voltage, the current in that branch ceases to flow, and the effective impedance of the device is raised. When the terminal voltage exceeds the highest battery voltage, the impedance becomes equal to $R_{0}$, the maximum value. The resulting currentvoltage characteristic is one of constantly increasing slope, as shown in curve B. By combining branches of each type, a characteristic may be achieved whose slope is alternately increasing and decreasing, provided it always remains positive. Curve C, Fig. 8, is typical.
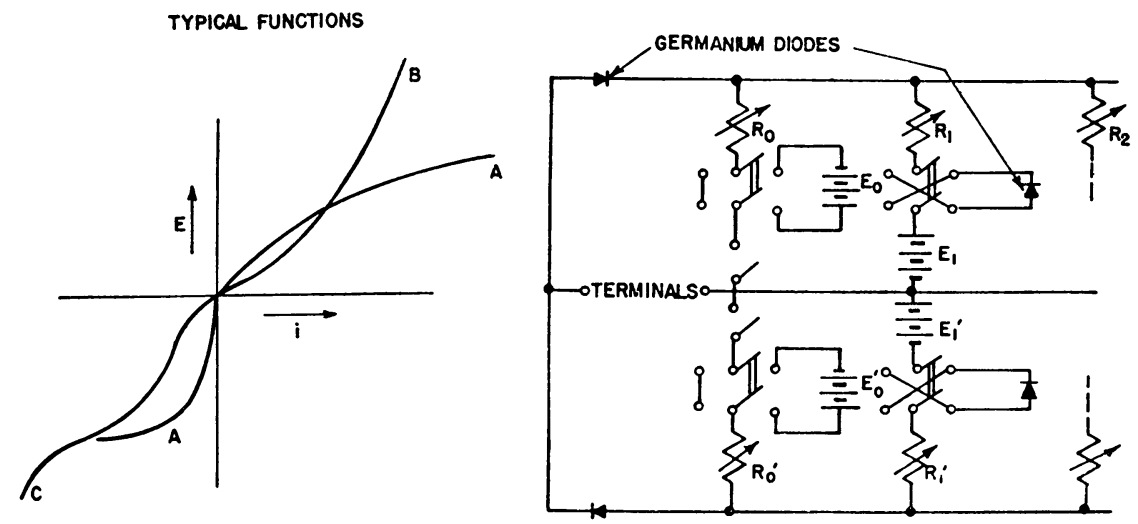

FIG. 8. Schematic Diagram-Dependent Variable Device Type 1.

It has been assumed that the voltage applied to the device is proportional to the dependent variable and that the current is proportional to an arbitrary function. If, however, the generator is driven by a current source, then the voltage across the device represents the arbitrary function. In this case, the type of characteristic will be reversed because of the interchange of axes (see Fig. 8).

The generator which is used with the computer has 22 parallel branches, 11 for positive voltages and 11 for negative voltages. A current-voltage characteristic composed of 22 straight-line segments can then be obtained. Such a characteristic can be used to approximate many typical functions with surprisingly good accuracy. Simpler and more specialized versions of the generator have also been developed in the form of abrupt current and voltage limiters.

The other arbitrary function generator (Type 2) is based upon a principle developed elsewhere. ${ }^{19,20}$ It employs a cathode-ray tube and photoelectric cell. With no signal, the electron beam is directed to one side of the tube. The photo-cell output is amplified and applied to the plates with the proper polarity to drive the beam to the other side. If an opaque template is placed on the screen of the tube, as shown in Fig. 9, the beam spot will not appear until the light reaching the photo-tube produces a large enough signal to deflect the beam above the edge of the template. 
The generator has several notable limitations. There is some distortion for which compensation is difficult, since the spot is not infinitely small and because the amplifier gain, the spot intensity, the height of the pattern, and the quiescent position all may vary from time to time. In addition, the halo on the screen contributes an appreciable signal, which may cause clipping of sharp, high peaks of the template, since the halo signal can be picked up on both sides of the peak with the spot completely hidden. Since the actual spot is on the rear of the cathode-ray screen while the observed pattern is on the front, there will be a third source of error in the resulting parallax. Careful proportioning of over-all height and width of the pattern, location of quiescent position, and adjustment of gain minimize these difficulties, but a definite accuracy limitation exists. The inability of the beam to rise or fall abruptly introduces another limitation. Thus, if the dependent variable passes rapidly through a region where the arbitrary function changes abruptly, the output will lag behind the ideal curve. This effect can be minimized by reducing the vertical scale of the template. Since the time lag is very small compared to the period of the highest frequency used on this computer, the effect on solutions is negligible. Despite these inherent limitations, it has been possible to develop a device that will form an arbitrary function of a dependent variable to within one per cent of the function's crest magnitude.

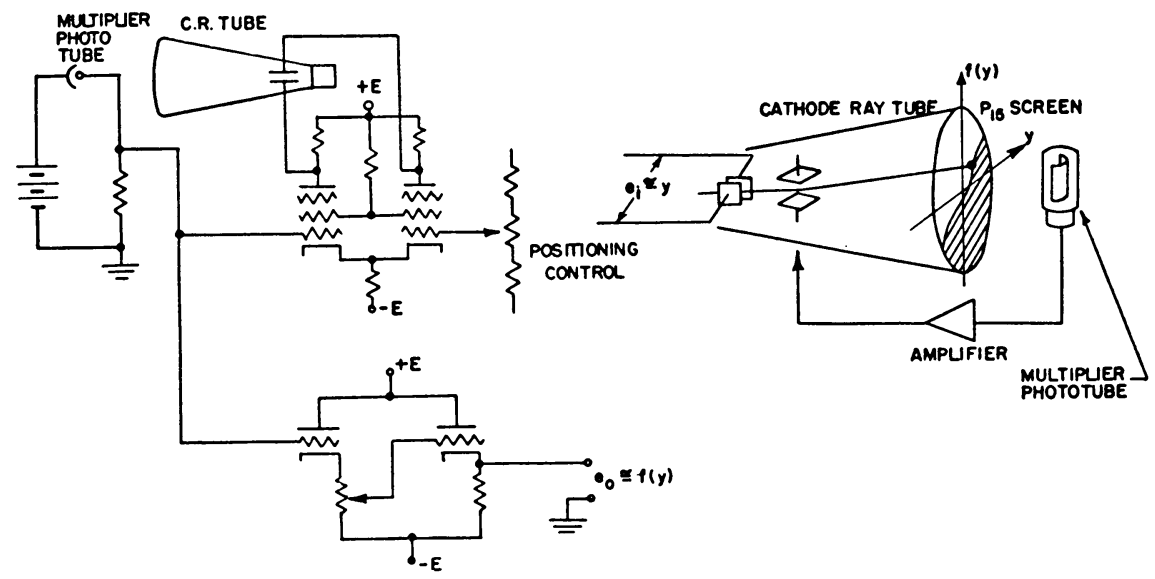

FIG. 9. Schematic Diagram of Arbitrary Forcing Function of Dependent Variable-Type 2.

Multiplier. The multiplication of two arbitrary voltages can be accomplished in many ways. The method which has been adopted for the CIT Computer uses double modulation followed by detection. ${ }^{7} \mathrm{~A}$ balanced modulator produces sidebands by modulating a carrier with one of the product terms. The resulting voltage is of the form $E_{1} \cos \left(\omega_{c} t\right)$. This voltage is used as a carrier for a second modulator which gives a sideband output, $E_{1} E_{2} \cos \left(\omega_{c} t\right)$. The carrier voltage is, of course, suppressed through use of a balanced modulator in both cases. Addition of a carrier voltage permits detection, with the output voltage proportional to the product, $E_{1} E_{2}$. The d-c voltage resulting from detection of the added carrier is balanced by detection of another carrier signal and subtraction of the outputs. For con- 
venience, a varistor bridge is used for the first modulator and vacuum tubes for the second modulator. Detection is accomplished with germanium diodes, thus minimizing the power supply requirements of the unit. Isolation between input and output is automatically supplied by the high-frequency transformers. One of the input voltages is not isolated from the system ground. If such isolation is required for both inputs, an additional isolator must be used. Care must be taken to minimize phase shift in the sidebands since this results in a phase shift of the detected signal. The first modulator, with untuned transformers, performs very well in this respect. Since tuned circuits are required in the second modulator for several reasons, the phase shift is minimized by over-coupling of the transformer to the plate circuit of the modulator. In the case of the multiplier, the accuracy is much better than the over-all one per cent requirement. The groups of multipliers and $\mathrm{d}-\mathrm{c}$ isolators in the computer are supplied by a common high-frequency generator, thus reducing the space required for each unit.

Present Fields of Application. Although the computer has been in active service for almost two years and has been applied to a wide range of problems in many fields of engineering and science, it is not yet possible to predict its complete range of usefulness. It may be more appropriate to merely list some of the more important types of problems for which it has been used.

In the field of servomechanisms and automatic control,9,10,12,14,17 the computer makes possible the rapid solution of control equations describing even the more comprehensive cases of missiles or autopilots with several degrees of freedom, and non-linearities in both the control system and the aerodynamic equations:

General analyses have been made of many simple and complex linear and non-linear ordinary differential equations pertaining to mechanical vibration analysis. ${ }^{8,11,16}$ Equations have been solved occurring in such fields as packaging of delicate apparatus, the investigation of the design and performance of turbine generators, short-circuit torques, airplane landing shock, acceleration through resonance, and earthquake shock effects on buildings.

A practical method has now been developed for solving accurately and rapidly the more general partial differential equations of elastic beams and plates. ${ }^{18}$ This facilitates the solution of the general types of transient beam and plate problems, as well as static loading and steady-state vibration problems.

The computer has been used for the solution of several linear thermal conductivity problems involving time and two space variables, and of nonlinear conductivity problems involving time and one space variable. Complex boundary conditions and heat generation within the media can be readily handled.

Other fields for which the California Institute of Technology Electric Analog Computer has so far been used include compressible fluid flow studies, complex electric circuit analysis, aircraft landing stability analysis, basic mechanical system backlash and non-linear friction analysis, rideability analysis of automobiles and railroad cars, and many others.

California Institute of Technology

G. D. McCANN

Pasadena, California 
${ }^{1}$ H. E. Criner, G. D. McCann, \& C. E. Warren, "A new device for the solution of transient-vibration problems by the method of electrical-mechanical analogy," Jn. Appl. Mech., v. 12, Sept. 1945, p. 135-141.

2 G. D. MCCANN \& H. E. CRINER, “Mechanical problems solved electrically,” Westinghouse Engin., v. 6, Mar. 1946, p. 48-56.

${ }^{3}$ G. D. MCCANN \& H. E. CRINER, "Solving complex problems by electrical analogy, part I," Machine Design, v. 17, Dec. 1945, p. 137-142.

${ }^{4}$ G. D. MCCANN \& H. E. CRINER, "Solving complex problems by electrical analogy, part II," Machine Design, v. 18, Feb. 1946, p. 129-132.

'G. D. MCCANN, "The mechanical-transients analyzer," Proceedings of the National Electronics Conference, Chicago, Ill., Oct. 1946, v. 2, p. 372-387.

'E. L. Harder \& G. D. MCCANn, "A large-scale general-purpose electric analog computer," AIEE, Trans., v. 67, 1948, p. 664-673.

${ }^{7}$ G. D. McCann, C. H. Wilts \& B. N. Locanthi, "Electronic techniques applied to analog methods of computation," IRE, Proc., v. 37, 1949, p. 954-961.

${ }^{8}$ G. D. MCCann, C. E. Warren, \& H. E. Criner, "Determination of transient shaft torques in turbine generators by means of the electrical-mechanical analogy," AIEE, Trans., v. 64,1945 , p. $51-56$.

9 G. D. McCann, S. W. Herwald, \& H. S. Kirschbaum, "Electrical analogy methods applied to servomechanism problems," AIEE, Trans., v. 65, 1946, p. 91-96, 515.

${ }^{10} \mathrm{G}$. D. MCCaNn \& S. W. Herwald, "Dimensionless analysis of servomechanisms by electrical analogy," AIEE, Trans., v. 65, 1946, p. 636-639, 1132; v. 66, 1947, p. 111-118.

${ }^{11} \mathrm{G}$. D. MCCANN \& J. M. KopPER, "Generalized vibration analysis by means of the mechanical-transients analyzer," Jn. Appl. Mech., v. 14, 1947, p. A127-A134.

${ }^{12} \mathrm{E}$. L. HARDER, "Solution of the general voltage regulator problem by electrical analogy," AIEE, Trans., v. 66, 1947, p. 815-825.

${ }_{13}$ G. D. MCCann, W. O. Osbon, \& H. S. Kirschbaum, "General analysis of speed regulators under impact loads," AIEE, Trans., v. 66, 1947, p. 1243-1252.

${ }_{14}$ G. D. McCanN, F. C. Lindvall, \& C. H. Wilts, "Effect of Coulomb friction on the performance of servomechanisms," AIEE, Trans., v. 67, 1948, pt. 1, p. 540-546.

${ }^{15} \mathrm{G}$. D. MCCANN \& C. H. WILTS, "Application of electric-analog computers to heattransfer and fluid-flow problems," Jn. Appl. Mech., v. 16, 1949, p. 247-258.

${ }^{16} \mathrm{G}$. D. MCCANN \& R. R. BenNetr, "Vibration of multifrequency systems during acceleration through critical speeds," Jn. Appl. Mech., v. 16, 1949 (in press).

${ }^{17}$ G. D. McCanN, C. H. Wilts, \& B. N. Locanthi, "Application of the Cal. Tech. Electric Analog Computer to non-linear mechanisms and servomechanisms," AIEE, Trans., 1949 (in press). Techn. Paper, no. 49-165.

${ }^{18} \mathrm{G}$. D. MCCANN \& R. H. MACNeal, "Beam vibration analysis with the electric analog computer," Jn. Appl. Mech., v. 16, 1949, p. A (in press).

19 D. M. MACKAY, "A high-speed electronic function generator," Nature, v. 159, Mar. 22, 1947, p. 406.

${ }^{20}$ D. J. Mynall, "Electrical analogue computing," Electronic Engin., London, v. 19, 1947 , p. 178-180, 214-217, 254-262, 283-285.

\section{TABLE}

Basic Elements of the California Institute of Technology Electric Analogue Computer

A. Ten Passive Circuit Elements Cabinets
1. 100 high " $Q$ " inductors
2. 20 high " $Q$ ", inductors
0 to 1.0 henrys in 0.001 henry steps
3. 100 resistors
4. 20 resistors
5. 60 capacitors
6. 40 capacitors
7. 10 capacitors
8. 25 special transformers*
0 to 1.50 henrys in 0.01 henry steps
0 to 7,000 ohms in $2 \mathrm{ohm}$ steps
0 to $500,000 \mathrm{ohms}$ in $200 \mathrm{ohm}$ steps
0 to $1.0 \mathrm{mfd}$ in $0.01 \mathrm{mfd}$ steps
0 to $4.0 \mathrm{mfd}$ in $0.01 \mathrm{mfd}$ steps
0 to $80 \mathrm{mfd}$ in $10 \mathrm{mfd}$ steps

B. Five Plugboards

1. Plugboards for all elements of two circuit elements cabinets

2. Receptacle patch-board for 500 element connections and 25 main busses

C. Two Forcing Functions Cabinets

1. 10 sinusoidal variable-frequency and phase-position-voltage sources (0 to 50 volts, one ampere, one ohm) 
2. 12 separate square wave voltage sources ( 0 to 12 volts, or one 72 volt source in 2 volt steps)

3. 5 arbitrary functions of independent variable

4. 5 arbitrary current sources (controllable from any voltage source)

\section{Two Metering Desks}

1. 3 dynamometer-type meters and 2 vacuum tube meters for metering sinusoidal current, voltage, power, etc.

2. 3 cathode-ray oscillographs for transient soiution (current, voltage, or charge)

3. 2 three-wire metering circuits (and selector system) for metering all passive circuit elements and source forcing functions

4. 2 manual metering selector circuits for 16 of 25 main busses

5. Circuits for connecting 36 synchronous switches to any of 26 main busses

\section{E. Amplifier Cabinets (portable)}

1. 5 negative gain ( 0 to 50 ) d-c amplifiers ( $15 \mathrm{ohm}$ impedance), each having an RC time-delay circuit for servo-problems

2. 15 positive gain (0 to 100$)$ d-c amplifiers with input and one ohm output impedance

3. $10 \mathrm{~d}-\mathrm{c}$ isolators and preamplifiers

F. Multiplier Cabinets (portable)

1. 10 multipliers with isolated inputs*

G. Arbitrary Functions of Dependent Variable (portable)

1. 3 voltage limiters

2. 2 current limiters

3. 1 arbitrary function of dependent variable-Type I

10 arbitrary functions of dependent variable-Type II

* Additional elements are contemplated. The Table lists only elements of the computer in its present operating form.

\section{RECENT MATHEMATICAL TABLES}

658[A-D, Q, R].-Italy, Istituto Idrografico della R. Marina, Tavole Logaritmiche a Cinque Cifre Decimali. Sixth ed. Genoa, 1941, xxii, 512. $19.7 \times 27.8 \mathrm{~cm}$. Full cloth, and gilt titles.

Contents: T. I (p. 1-57).-Mantissae of logarithms and cologarithms of numbers from 1 to 10080 ; T. II (p. 60-179)-Logarithms of the absolute values of the trigonometric functions $0\left(1^{\prime \prime}\right) 2^{\circ}$; T. III (p. 182-361)-Logarithms of the absolute values of the trigonometric functions $0\left(15^{\prime \prime}\right) 90^{\circ}$; T. IV (p. 364-382)-Addition and subtraction logarithms; T. V (p. 384-428)-Values of the natural trigonometric functions $0\left(1^{\prime}\right) 90^{\circ}$ or $0\left(4^{s}\right) 6^{h}$; T. VI-XIII (430-464)-Conversion of common logarithms to natural, and vice versa; $n^{2}, n^{3}, n^{\mathbf{k}}, n^{\mathbf{\}}}, \ln n$, $1000 n^{-1}, \pi n, \frac{1}{4} \pi n^{2}, n=0(1) 1020$; table of arcs, altitude of sector, chord for $0\left(1^{\circ}\right) 180^{\circ}$, also arcs corresponding to $0\left(1^{\prime}\right) 60^{\prime}, 0\left(1^{\prime \prime}\right) 60^{\prime \prime}$; conversion of centesimal grades, minutes and seconds into sexagesimals; conversion of arcs into time and vice versa; conversion of sexagesimal minutes and seconds into decimals of degrees, and vice versa; geodetic elements relative to the international ellipsoid; values of international normal gravity, $g$, for $\phi$ (geogr. latitude) $=0\left(15^{\prime}\right) 90^{\circ}$. Constants and units of measure (p. 467-480). Formulae (p. 483-512) -algebra, plane analytic geometry, differential and integral calculus, plane and spherical trigonometry, series, interpolation, astronomy, geodesy, least squares.

Editorial Note-The first edition of this work was published in 1913; the second, 1916; third, 1922; fourth, 1930; fifth, 1937. There was a "nuova edizione, ridotta," 128 p., in 1939 , and a copy may be seen in Library of Congress. 